\title{
On the minimum mass of neutron stars
}

\author{
Yudai Suwa, ${ }^{1,2 \star}$ Takashi Yoshida, ${ }^{3} \dagger$ Masaru Shibata,,${ }^{1,4}$ Hideyuki Umeda, ${ }^{3}$ \\ and Koh Takahashi ${ }^{5}$ \\ ${ }^{1}$ Center for Gravitational Physics, Yukawa Institute for Theoretical Physics, Kyoto University, Kyoto 606-8502, Japan \\ ${ }^{2}$ Department of Astrophysics and Atmospheric Sciences, Faculty of Science, Kyoto Sangyo University, Kyoto 603-8555, Japan \\ ${ }^{3}$ Department of Astronomy, Graduate School of Science, University of Tokyo, Tokyo 113-0033, Japan \\ ${ }^{4}$ Max Planck Institute for Gravitational Physics (Albert Einstein Institute), Am Mühlenberg 1, Potsdam-Golm, 14476, Germany \\ ${ }^{5}$ Argelander-Institute für Astronomie, Universitaüt Bonn, D-53121 Bonn, Germany
}

Accepted XXX. Received YYY; in original form ZZZ

\begin{abstract}
We investigate the remnant neutron star mass, in particular the minimum mass. We perform advanced stellar evolution calculations and neutrino-radiation hydrodynamics simulations for core-collapse supernova explosions based on the standard astrophysical scenarios. We find that from low-mass carbon-oxygen cores which form sufficiently massive iron cores that eventually collapse, the remnant neutron stars after supernova explosion are as light as $1.17 \mathrm{M}_{\odot}$, which is compatible with the lowest mass of the neutron star precisely measured in a binary system of PSR J0453+1559.
\end{abstract}

Key words: binaries: general - stars: massive - stars: neutron - supernovae: general

\section{INTRODUCTION}

Mass of neutron stars (NSs) is one of the most important observables to probe high-density nuclear physics. In particular, the maximum mass of NSs gives a stringent constraint on the nuclear physics above the saturation density. The current largest mass is $\approx 2 \mathrm{M}_{\odot}$ (Demorest et al. 2010; Antoniadis et al. 2013), which means that hypothetical nuclear equations of state having maximum NS mass smaller than $2 \mathrm{M}_{\odot}$ are excluded.

Neutron star mass has a broad distribution. A precise measurement is possible for a binary system which contains at least one pulsar (Özel \& Freire 2016). Recently, the first asymmetric system of double neutron stars, PSR J0453+1559, was discovered (Martinez et al. 2015). Secondary NS's mass is much smaller than a canonical mass, that is, $1.174 \pm 0.004 \mathrm{M}_{\odot}$. A corresponding baryonic mass of this $\mathrm{NS}$ is $\approx 1.28 \mathrm{M}_{\odot}$, which is remarkably smaller than a typical mass of Fe cores, i.e. 1.3-1.6 $\mathrm{M}_{\odot}$ (e.g., Sukhbold et al. 2018). From this observation, a natural question arises: Is it possible to form such a low-mass NS with the standard scenario of supernova (SN) explosion?

Although the explosion mechanism of core-collapse $\mathrm{SNe}$ is still unclear, there is the standard paradigm called neutrino-driven explosion (Bethe \& Wilson 1985), in which neutrinos produced in the vicinity of newly-born NS heat up

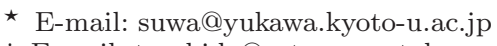

$†$ E-mail: tyoshida@astron.s.u-tokyo.ac.jp the post-shock material. Aided by the multi-dimensional hydrodynamic effects, e.g. convection and standing accretion shock instability, the explosions driven by neutrino heating have been reported in the past decade (see Burrows 2013; Janka et al. 2016, for recent reviews and references therein). ${ }^{1}$ In this work, we assume that $\mathrm{SN}$ explosion is driven by the neutrino heating mechanism.

Producing a low-mass NS is also an issue of the massive star evolution. Evolved stars with a carbonoxygen $(\mathrm{CO})$ core heavier than $\sim 1.37 \mathrm{M}_{\odot}$ have a possibility of SN explosion and NS formation (e.g. Nomoto 1987). Single stars with a zero-age-main-sequence (ZAMS) mass of $\sim 8-12 \mathrm{M}_{\odot}$ have a path to electron-capture (EC) SNe (Nomoto 1987; Takahashi et al. 2013) or core-collapse SNe from a low-mass Fe core (e.g. Woosley et al. 1980; Nomoto 1984; Nomoto \& Hashimoto 1988; Umeda et al. 2012; Woosley \& Heger 2015). Studies on a large number of progenitors suggest that these stars would produce low-mass NSs (Ugliano et al. 2012; Ertl et al. 2016; Sukhbold et al. 2016). Note that fall-back accretion would increase NS mass for stars with massive envelope, and thus, the formation mechanism of the low-mass NS is not trivial.

In close-binary systems, ultra-stripped $\mathrm{SN}$ is a possible path to produce a low-mass NS (e.g. Tauris et al. 2013, 2015; Suwa et al. 2015; Moriya et al. 2017; Müller et al. 2018).

\footnotetext{
1 Note that the current simulations cannot account for the observed explosion energy and nickel amount for canonical supernovae (e.g. Suwa et al. 2017).
} 
Most of $\mathrm{H}$ and $\mathrm{He}$ envelope for these stars could be lost during their evolution by the close binary interactions. The explosion process of ultra-stripped SNe, especially the mass accretion history onto the proto-neutron star (PNS), would be considerably different from those of single stars. Thus, in this paper, we investigate NS formation in ultra-stripped SNe.

In the following, we investigate the path to produce such a low-mass NS based on standard methods of stellar evolution and supernova explosion simulations. As in the previous works (Suwa et al. 2015; Yoshida et al. 2017), which explored the evolution of massive stars whose envelope is supposed to be significantly stripped, we systematically study the dependence of the stellar evolution on the initial $\mathrm{CO}$ core mass as parametric manner. By extending the previous works toward lower $\mathrm{CO}$ core mass, we evaluate the minimum mass of an NS determined from the stellar evolution and demonstrate that a low-mass NS like PSR J0453+1559 can be produced in a standard scenario of binary NS formation. The paper is organized as follows. Section 2 describes our stellar evolutionary calculations, in particular focusing on the consequent core masses. Section 3 gives estimates of Chandrasekhar mass which depends on the profiles of the electron fraction and entropy as well as the iron core mass. The numerical method of subsequent hydrodynamics simulations and the results are presented in Section 4. In Section 5 we discuss differences of $\mathrm{EC} \mathrm{SNe} \mathrm{from} \mathrm{Fe-core} \mathrm{forming}$ core-collapse SNe in ultra-stripped SNe. We summarize our results in Section 6 .

\section{CO CORE MODELS}

We calculate the evolution of nine $\mathrm{CO}$ cores with the mass of 1.35-1.45 $\mathrm{M}_{\odot}$ from the central $\mathrm{C}$ burning. We denote the $\mathrm{CO}$ core model with the mass of $\mathrm{x} . \mathrm{yz} \mathrm{M}_{\odot}$ as COxyz model. These $\mathrm{CO}$ cores correspond to the hypothetically secondary star in the close binary system, which is supposed to lose their $\mathrm{H}$ and He-rich envelope during the binary evolution with a primary NS. The evolution calculations are conducted with the stellar evolution code used in Suwa et al. (2015) (see also Yoshida et al. 2017). We include the Coulomb corrections for the weak interaction rates which depend on the temperature and the electron number density (Toki et al. 2013). We take about 1000 mass zones in each model.

The nuclear reaction network of 300 species of nuclei is adopted. The adopted nuclear species are as follows; ${ }^{1} \mathrm{n}$, ${ }^{1-3} \mathrm{H},{ }^{3,4} \mathrm{He},{ }^{6,7} \mathrm{Li},{ }^{7,9} \mathrm{Be},{ }^{8,10,11} \mathrm{~B},{ }^{11-16} \mathrm{C},{ }^{13-18} \mathrm{~N},{ }^{14-20} \mathrm{O}$, ${ }^{17-22} \mathrm{~F},{ }^{18-24} \mathrm{Ne},{ }^{21-26} \mathrm{Na},{ }^{22-28} \mathrm{Mg},{ }^{27-32} \mathrm{Si},{ }^{27-34} \mathrm{P},{ }^{30-37} \mathrm{~S}$, ${ }^{32-38} \mathrm{Cl},{ }^{34-43} \mathrm{Ar},{ }^{36-45} \mathrm{~K},{ }^{38-48} \mathrm{Ca},{ }^{40-49} \mathrm{Sc},{ }^{42-51} \mathrm{Ti},{ }^{44-53} \mathrm{~V}$, ${ }^{46-55} \mathrm{Cr},{ }^{48-57} \mathrm{Mn},{ }^{50-61} \mathrm{Fe},{ }^{51-62} \mathrm{Co},{ }^{54-66} \mathrm{Ni},{ }^{54-66} \mathrm{Cu}$, ${ }^{59-71} \mathrm{Zn},{ }^{61-71} \mathrm{Ga},{ }^{63-75} \mathrm{Ge},{ }^{65-76} \mathrm{As},{ }^{67-77} \mathrm{Se},{ }^{70-79} \mathrm{Br}$. The isomeric state of ${ }^{26} \mathrm{Al}$ is taken into account. We set the initial chemical compositions of the CO cores as evaluated in Suwa et al. (2015). The initial chemical compositions of the $\mathrm{CO}$ cores are evaluated using the evolution of $\mathrm{H}$ and $\mathrm{He}$ burnings. The mass fractions of $\mathrm{C}$ and $\mathrm{O}$ of these models are assumed to be 0.360 and 0.611 , respectively. We have confirmed that properties of ultra-stripped SN progenitors do not strongly depend on the C/O ratio in Suwa et al. (2015). Detailed evolution properties are described in Appendix A.

We evaluate that the mass range of single stars at ZAMS that form a $\mathrm{CO}$ core with $1.37-1.45 \mathrm{M}_{\odot}$ is $9.25-9.75 \mathrm{M}_{\odot}$ using the same manner in Suwa et al. (2015). The evolution of stars that have the similar range of $\mathrm{CO}$ core was investigated in Woosley \& Heger (2015). We compare the mass range of our study with their result. From Table 1 of Woosley \& Heger (2015), the ZAMS mass range of single stars that form a $\mathrm{CO}$ core with $1.35-1.45 \mathrm{M}_{\odot}$ is about 8.8-9.3 $\mathrm{M}_{\odot}$. The relation between the total ZAMS mass and the CO core mass depends mainly on the overshoot treatment of convective layers. Strong overshoot gives a large $\mathrm{CO}$ core mass for a given ZAMS mass. Thus, the overshoot effect during the $\mathrm{H}$ and $\mathrm{He}$ burnings in our models would be weaker than their single star models. The overshoot is actually constrained from observations of mainsequence stars. We use the overshoot parameter with the description in Takahashi et al. (2014) and the parameter value of $f_{\mathrm{ov}}=0.015$, which reproduces the main-sequence band width observed for $\mathrm{AB}$ type stars in open clusters in the Galaxy (Maeder \& Meynet 1989).

We obtain a critical mass for Ne ignition in the $\mathrm{CO}$ core models through the evolution calculations. The $\mathrm{CO}$ core models with $M_{\mathrm{CO}} \geq 1.36 \mathrm{M}_{\odot}$ start off-center Ne burning. The evolution of the $\mathrm{CO}$ cores with $M_{\mathrm{CO}} \geq 1.37 \mathrm{M}_{\odot}$ is calculated until the central density reaches $\sim 10^{10} \mathrm{~g} \mathrm{~cm}^{-3}$. These stars form an Fe core. For CO136 model, the calculation is stopped when the density becomes $10^{9.5} \mathrm{~g} \mathrm{~cm}^{-3}$ and the Fe layer is formed. Although we did not calculate further evolution, we expect that this model will form an Fe core and will collapse. On the other hand, CO135 model does not cause $\mathrm{Ne}$ ignition. The temperature rises to $1.2 \times 10^{9} \mathrm{~K}$ at 0.97 $\mathrm{M}_{\odot}$ after the $\mathrm{C}$ shell burning and the temperature starts to decrease. We continue the calculation until the central density becomes $10^{9.1} \mathrm{~g} \mathrm{~cm}^{-3}$ and the central temperature becomes below $10^{8.15} \mathrm{~K}$. Thus, a critical mass for Ne ignition is $\sim 1.36 \mathrm{M}_{\odot}$ in our CO core models. Nomoto (1984) showed a critical mass of $1.37 \mathrm{M}_{\odot}$ for Ne ignition from the evolution calculation of pure Ne star models. Recently, Schwab et al. (2016) showed a critical mass of $1.35 \mathrm{M}_{\odot}$ for Ne ignition of pure Ne star models using stellar evolution code MESA (e.g. Paxton et al. 2015). Although our model is not an Ne star model and some input physics are different, a critical mass of $\mathrm{CO}$ star models for $\mathrm{Ne}$ ignition in our study is close to criteria in these studies.

The main properties of the $\mathrm{CO}$ core models with $M_{\mathrm{CO}} \geq$ $1.37 \mathrm{M}_{\odot}$ are listed in Table 1 . The Fe core mass at the last moment of the stellar evolution is evaluated using three different criteria: the entropy $\left(s \leq 3\right.$ in units of $k_{B}$ per baryon, where $k_{B}$ is Boltzmann's constant), the electron fraction $\left(Y_{e} \leq 0.495\right)$, and the chemical composition $(X(" \mathrm{Fe}$ ") $>X$ ("Si"), where $X(" \mathrm{Fe} ")$ and $X$ ("Si") denote the mass fractions of Fe-peak elements $(Z \geq 22)$ and intermediate elements ( $14 \leq Z \leq 21)$ with $Z$ being atomic number). We find that the Fe core mass is about $\sim 1.3 \mathrm{M}_{\odot}$ in these models. This mass is roughly determined by the arrival position of the convection during the O-shell burning after the off-center $\mathrm{O}$ burning. More details are described in the next section. 
Table 1. Model summary.

\begin{tabular}{|c|c|c|c|c|c|c|c|}
\hline Model & $\begin{array}{l}M_{\mathrm{CO}} \\
\left(\mathrm{M}_{\odot}\right)\end{array}$ & $\begin{array}{c}M_{\text {ZAMS }} \\
\left(\mathrm{M}_{\odot}\right)\end{array}$ & $\begin{array}{l}M_{S=3} \\
\left(\mathrm{M}_{\odot}\right)\end{array}$ & $\begin{array}{c}M_{Y_{e}=0.495} \\
\left(\mathrm{M}_{\odot}\right)\end{array}$ & $\begin{array}{l}M_{\mathrm{Fe}} \\
\left(\mathrm{M}_{\odot}\right)\end{array}$ & $\begin{array}{c}M_{\mathrm{NS}, \text { bary }} \\
\left(\mathrm{M}_{\odot}\right)\end{array}$ & $\begin{array}{c}M_{\mathrm{NS}, \text { grav }} \\
\left(\mathrm{M}_{\odot}\right)\end{array}$ \\
\hline CO137 & 1.37 & 9.35 & 1.347 & 1.314 & 1.280 & 1.289 & 1.174 \\
\hline CO138 & 1.38 & 9.40 & 1.349 & 1.316 & 1.274 & 1.296 & 1.179 \\
\hline CO139 & 1.39 & 9.45 & 1.350 & 1.320 & 1.258 & 1.302 & 1.184 \\
\hline CO140 & 1.40 & 9.50 & 1.305 & 1.302 & 1.296 & 1.298 & 1.181 \\
\hline CO142 & 1.42 & 9.60 & 1.284 & 1.280 & 1.265 & 1.287 & 1.172 \\
\hline CO144 & 1.44 & 9.70 & 1.275 & 1.219 & 1.234 & 1.319 & 1.198 \\
\hline CO145 & 1.45 & 9.75 & 1.362 & 1.270 & 1.277 & 1.376 & 1.245 \\
\hline
\end{tabular}

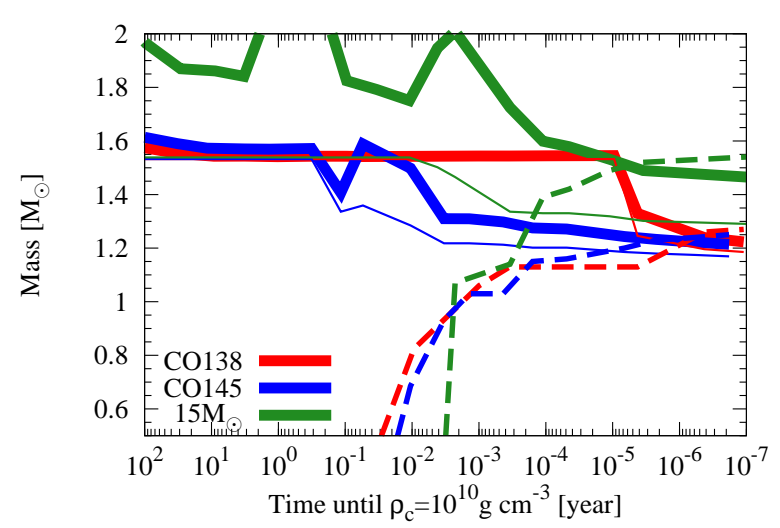

Figure 1. Time evolution of modified Chandrasekhar mass (see Eq. (2)) (solid curves) and Fe core mass (dashed curves), for models CO138 (red), CO145 (blue), and a model with $M_{\text {ZAMS }}=15$ $\mathrm{M}_{\odot}$ for comparison. In the thin solid curves, the thermal correction is not taken into account, while the thick solid curves include the correction.

\section{CORE MASSES}

In this section, we discuss the evolution of the core and its critical mass, above which the core becomes unstable against the self-gravity, to investigate the condition of core collapse.

Figure 1 shows time evolution of modified Chandrasekhar mass (Baron \& Cooperstein 1990; Timmes et al. 1996) and the Fe core mass. In the modified Chandrasekhar mass, the finite temperature correction is taken into account as follows,

$M_{\mathrm{Ch}}=M_{\mathrm{Ch} 0}\left[1+\left(\frac{s_{e}}{\pi Y_{e}}\right)^{2}\right]$,

where $M_{\mathrm{Ch} 0}=1.46 \mathrm{M}_{\odot}\left(Y_{e} / 0.5\right)^{2}$ is Chandrasekhar mass without finite temperature correction and $s_{e}$ is the electronic entropy per baryon. The entropy is typically given as $s_{e}=0.56\left(Y_{e} / 0.5\right)^{2 / 3}(T / 1 \mathrm{MeV})\left(\rho / 10^{10} \mathrm{~cm}^{-3}\right)^{-1 / 3} k_{B}$ baryon $^{-1}$ with $\rho$ and $T$ being the density and temperature (Baron \& Cooperstein 1990). In this paper, we derive an expression in which $Y_{e}$ distribution is taken into account (see Appendix B) as

$M_{\mathrm{Ch}}=1.09 M_{\odot}\left(\frac{Y_{e, c}}{0.42}\right)^{2}\left[1+\left(\frac{s_{e, c}}{\pi Y_{e, c}}\right)^{2}\right]$,

where $Y_{e, c}$ and $s_{e, c}$ are the central values of $Y_{e}$ and $s_{e}$. Figure 1 shows that $M_{\mathrm{Ch}}$ decreases with time just prior to the onset of collapse because the electron capture and neutrino cooling reduces $Y_{e}$ and entropy, respectively. The Fe core mass (the outermost mass coordinate that has an Fe mass fraction larger than 0.5), on the other hand, increases by shell burning of Si. The collapse takes place when its mass increases over $M_{\mathrm{Ch}}$. If we neglect the finite temperature correction, the core mass exceeds $M_{\mathrm{Ch}}$ much earlier. We also plot a canonical single star evolution (for $M_{\text {ZAMS }}=15 \mathrm{M}_{\odot}$ ) in this figure, which has a larger correction of the finite temperature effect than the present $\mathrm{CO}$ core models so that it has a larger core mass when it collapses.

In Figure 2, the density, the electron fraction, and the entropy profile when the central density becomes $10^{10} \mathrm{~g}$ $\mathrm{cm}^{-3}$ (approximately at the onset of core collapse) are presented. The central part $\left(M \lesssim 1.2 \mathrm{M}_{\odot}\right)$ is rather similar among all the models shown here, but the outer part depends strongly on the value of $M_{\mathrm{CO}}$. This difference stems primarily from the extension of strong O-shell burning after the off-center $\mathrm{O}$ burning. The decrease in the electron fraction of this region is occurred by the O-shell burning. In the models with $M_{\mathrm{CO}}<1.44 \mathrm{M}_{\odot}$, the convective region of the O-shell burning extends to $\sim 1.2 \mathrm{M}_{\odot}$ or more (see panels $(e)$ and $(f)$ of Fig. A2 for CO142 model). On the other hand, the convection does not reach $\sim 1.2 \mathrm{M}_{\odot}$ for $\mathrm{CO} 144$ and CO145 models. The electron fraction for the mass coordinate in the range of $1.20-1.27 \mathrm{M}_{\odot}$ for CO145 model is reduced by the following O-shell burning. For CO144 model, the off-center Si burning starts just after the O-shell burning sets in followed by the off-center $\mathrm{O}$ burning. The region outside the convective Si layer expands and the O-shell burning is ceased. Thus, the electron fraction is not reduced for $M_{r} \gtrsim 1.2 \mathrm{M}_{\odot}$ for CO144 model.

\section{EXPLOSION SIMULATIONS}

Starting from the progenitor models described in the previous section, we perform two-dimensional neutrinoradiation hydrodynamics simulations as of Suwa et al. (2015); Yoshida et al. (2017). In the previous works, we employed little bit heavier CO cores as from 1.45 to $2 \mathrm{M}_{\odot}$ to make comparison with Tauris et al. (2013), in which a star with $1.5 \mathrm{M}_{\odot}$ was investigated to account for a candidate of the ultra-stripped supernova SN 2005ek. This work, on the other hand, is more interested in the final mass of an NS so that progenitor models with a lower mass range are explored.

The numerical method is the same as Suwa et al. (2015), in which two-dimensional hydrodynamics equa- 


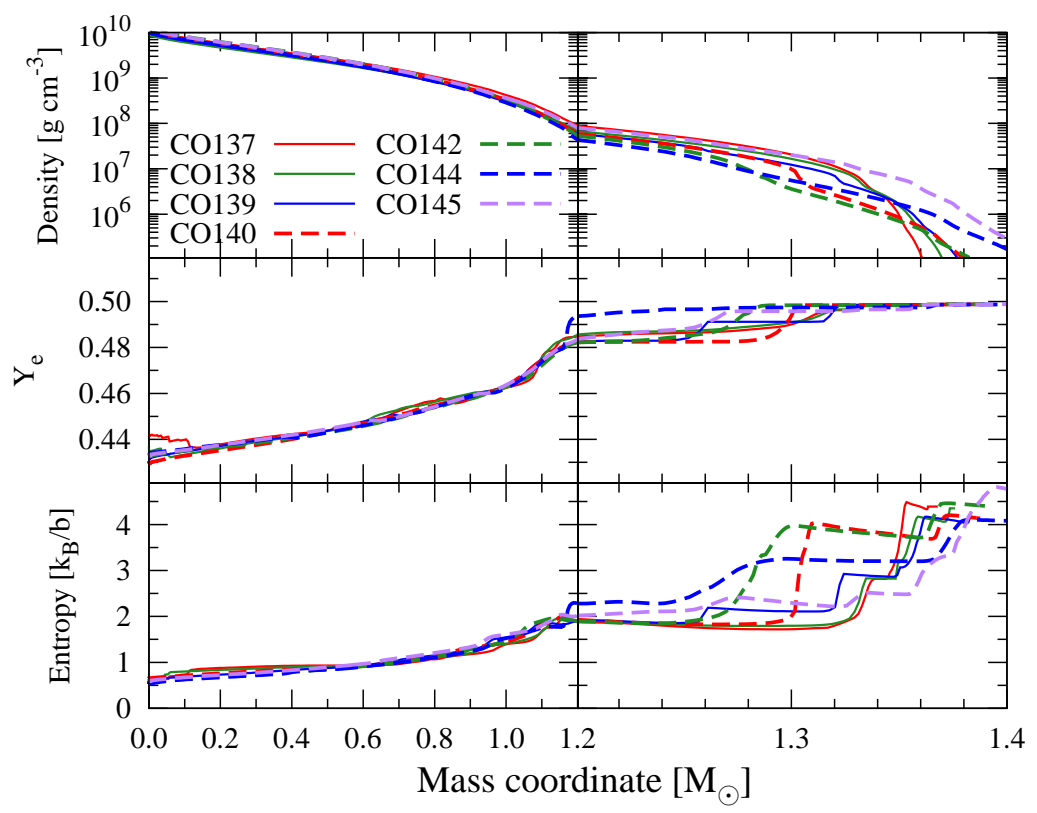

Figure 2. Density (top), electron fraction (middle), and entropy (bottom) structure as functions of mass coordinate for investigated models.

tions are solved as well as neutrino-radiation transfer equation with isotropic diffusion source approximation (IDSA) (Liebendörfer et al. 2009; Suwa et al. 2010). Rayby-ray plus approximation (Buras et al. 2006) is used to treat the multi-dimensional transfer with a spherically symmetric solver. The nuclear equation of state from Lattimer \& Swesty (1991) with incompressibility parameter $K=220 \mathrm{MeV}$ is employed.

The hydrodynamic properties are the same as those found in Suwa et al. (2015). After the onset of core collapse, it takes $O(100) \mathrm{ms}$ until an NS forms, depending on progenitor models $(403,714,1164,1022,1399,807$, and 698 ms for CO137, CO138, CO139, CO140, CO142, CO144, and CO145, respectively). They all result in explosions aided by convection in two-dimensional simulations. The explosion sets in when the density jump between the interface of $\mathrm{Si}$ and $\mathrm{Si} / \mathrm{O}$ layers accretes onto the shock and the ram pressure above the shock decreases much faster than the thermal pressure below the shock (see e.g., Suwa et al. 2016; Summa et al. 2016). Therefore, in the zeroth order approximation, the NS mass is determined by the mass coordinate of the interface (see also Sukhbold et al. 2018).

The consequent NS masses are shown in Table 1. We calculate the gravitational mass of NSs from the baryonic mass with Eq. (35) of Lattimer \& Prakash (2001). ${ }^{2}$ Note that the observable in binary pulsar systems is the gravita-

2 The mass decrease due to the binding energy of an NS is given as $\Delta M=M_{\mathrm{NS} \text {, bary }}-M_{\mathrm{NS} \text {, grav }}=0.084 \mathrm{M}_{\odot}\left(M_{\mathrm{NS} \text {, grav }} / \mathrm{M}_{\odot}\right)^{2}$ in this equation. The overall factor depends on nuclear equation of state, roughly $0.06-0.1$ for the mass range we are interested in (see Figure 8 of Lattimer \& Prakash 2001). This means that for $M_{\mathrm{NS}, \text { grav }}=1.17 \mathrm{M}_{\odot}$, the corresponding baryonic mass is 1.25 $1.31 \mathrm{M}_{\odot}$. Note that the equation of state used in this study (Lattimer \& Swesty 1991) would give $\sim 0.07$, but we use 0.084 for simplicity. tional mass. It is seen from this table that the gravitational mass of an NS can be as small as $1.17 \mathrm{M}_{\odot}$, which is consistent with the current observation of the smallest NS mass measured precisely.

\section{COMPARISON WITH ELECTRON-CAPTURE SUPERNOVAE}

The evolution of massive stars toward EC SN in the binary system with an NS has been discussed in Tauris et al. (2015). We suppose that an ultra-stripped EC SN may occur for a $\mathrm{CO}$ core with the mass less than $1.36 \mathrm{M}_{\odot}$ just after the $\mathrm{C}$ burning. An EC SN occurs when a $\mathrm{CO}$ core reaches the Chandrasekhar mass by the CO-core growth. In this case, it is important to clarify how the CO-core grows through the He-shell burning. In the case of a single star, the ONe-core mass of an EC SN progenitor is less than $\sim 1.37 \mathrm{M}_{\odot}$ after the C burning (e.g. Nomoto 1987; Takahashi et al. 2013). The ONe core mass increases to the modified Chandrasekhar mass through the He shell burning during the evolution of the super asymptotic giant branch (AGB) star. In an He star in the binary system, there is no H-rich envelope and, thus, AGB phase does not occur. For such a situation, the CO core growth depends on the efficiencies of the He shell burning and the mass loss of the He envelope. Because the mass loss process in the binary system is not fully understood and long-time calculation of the CO-core growth through the He-shell burning is required, it is difficult to evaluate whether a $\mathrm{CO}$ core less than $1.36 \mathrm{M}_{\odot}$ in the binary system grows up to the critical mass and explodes as an ultrastripped EC SN. The mass range of CO cores for EC SNe was recently discussed for the primary stars in binary system (Poelarends et al. 2017; Siess \& Lebreuilly 2018). However, the mass range would be still model dependent and, thus, we are not sure whether the binary evolution affects the mass 


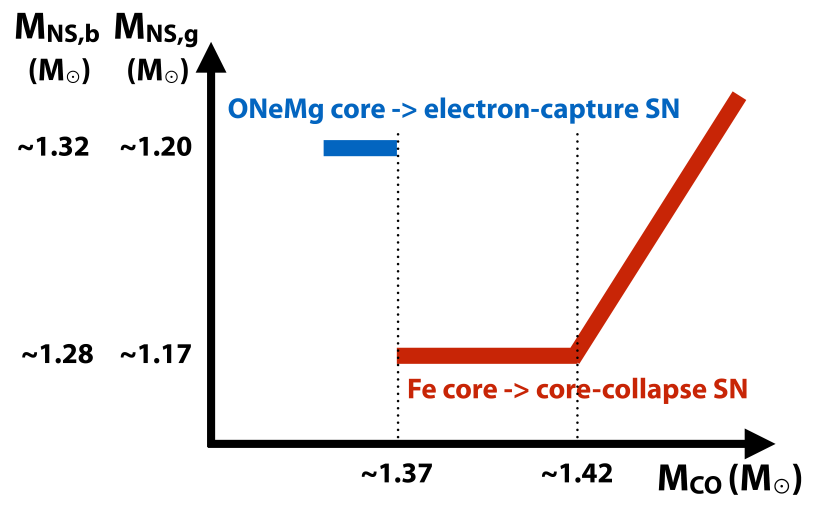

Figure 3. Rough relation between NS mass and CO core mass. Two numbers in NS mass are baryonic mass $\left(M_{\mathrm{NS}, \mathrm{b}}\right.$; left) and gravitational mass $\left(M_{\mathrm{NS}, \mathrm{g}}\right.$; right), respectively. For $M_{\mathrm{CO}} \lesssim 1.37 \mathrm{M}_{\odot}$, the electron-capture SN would be produced and the consequent NS mass would be $\sim 1.37 \mathrm{M}_{\odot}$ and $1.24 \mathrm{M}_{\odot}$ in baryonic mass and gravitational mass. For $M_{\mathrm{CO}} \gtrsim 1.37 \mathrm{M}_{\odot}$, the corecollapse SN would be produced and because of its lower-mass Fe core the consequent mass of NS is smaller than models which produce EC SN.

range of $\mathrm{CO}$ cores of EC SN progenitors. The investigation of the growth of the ONe core with thin He layer is important to clarify the possibility of the evolutionary path to an EC SN in close binary systems.

How is low-mass NS formed from EC SN progenitors and ultra-stripped SN progenitors that have a low-mass $\mathrm{Fe}$ core? In the case of $\mathrm{EC} \mathrm{SNe,} \mathrm{the} \mathrm{electron} \mathrm{fraction} \mathrm{in} \mathrm{the}$ ONe core is $\sim 0.48$ at the central Ne ignition (Takahashi et al. 2013, 2018). The electron fraction in the ONe core scarcely decreases until the central Ne ignition. On the other hand, progenitors of ultra-stripped SNe cause electron captures during their evolution and, as a result, the electron fraction of the Fe core decreases. The electron fraction at the center is $\sim 0.43$ when the central density becomes $\sim 10^{10} \mathrm{~g}$ $\mathrm{cm}^{-3}$ in the collapsed models in this paper. Thus, the Chandrasekhar mass of EC SN progenitors is heavier than that of the progenitors that have a low-mass Fe core of ultrastripped SNe. The hydrodynamical simulation in our previous study (Yoshida et al. 2017) showed that the baryon mass of the PNS of an EC SN is $1.32 \mathrm{M}_{\odot}$. This mass is higher than the baryon mass range of the NSs formed from CO137-CO144 models.

\section{SUMMARY}

In this paper, we investigated the minimum mass of NSs based on astrophysical scenario, i.e., stellar collapse and supernova explosion. We calculated the evolution of the $\mathrm{CO}$ cores with mass $1.35-1.45 \mathrm{M}_{\odot}$. The stars with the CO-core mass higher than $1.36 \mathrm{M}_{\odot}$ form an Fe core after off-center $\mathrm{Ne}, \mathrm{O}$, and Si-burnings. We found that low-mass CO cores, which eventually form an Fe core and subsequently collapse, could result in an NS with mass $\sim 1.17 \mathrm{M}_{\odot}$, which is the lowest mass of NSs precisely measured.

According to our stellar evolution simulations, the minimum mass of CO cores that produce an Fe core is $\sim 1.37 \mathrm{M}_{\odot}$ and below this value ONe core is formed, which would lead to EC SN instead. Because of its higher $Y_{e}$, an EC SN would produce more massive NSs than a core-collapse SN from the Fe core. Therefore, the minimum mass of NSs is expected to be determined by the core-collapse $\mathrm{SN}$ of a low-mass $\mathrm{CO}$ core (see Figure 3).

The range of the Fe core mass for single stars of initial mass 9-10 $\mathrm{M}_{\odot}$ in Woosley \& Heger (2015) is similar or smaller than ultra-stripped SN progenitors. Thus, the lowest-mass NSs may also be formed from the collapse of low-mass Fe cores. In the case of single stars, however, the reverse shock is produced when the shock wave arrives at the interface of the $\mathrm{H}$-rich envelope. Then, the fall-back material accretes onto the collapsed core and increases NS mass. In the case of ultra-stripped $\mathrm{SNe}$, on the other hand, the fallback will be negligible because of very thin He layer and no H-rich envelope. Due to the same reason, the primary NS generated by the first SN explosion (not ultra-stripped SN) in binary systems would be incompatible to the light NS in PSR J0453+1559. This is because the secondary star would supply mass to the NS during its giant phase and increase the NS mass. Thus, low-mass Fe cores in the progenitors of ultra-stripped $\mathrm{SNe}$, which are conjectured second explosions in close-binary systems, would be more favorable to form lowest-mass NSs.

\section{ACKNOWLEDGEMENTS}

The numerical computations in this study were partly carried out on XC30 at CfCA in NAOJ and XC40 at YITP in Kyoto University. This work was supported in part by the Grant-in-Aid for Scientific Research (Nos. 16H00869, 16H02183, 16K17665, 17H01130, 17H02864, 17H06364, 18H04586, 18H05437, 26400271, 26104007), MEXT as "Priority Issue on Post-K computer" (Elucidation of the Fundamental Laws and Evolution of the Universe) and a post $\mathrm{K}$ project (No. 9) of the Japanese MEXT. TY thanks Thomas M. Tauris, Nobert Langer, and Takashi J. Moriya for discussion on the advanced evolution of light $\mathrm{CO}$ core and a hospitality during short stays in Argelander-Institute für Astronomie, Universität Bonn in 2015 and 2018. KT is supported by Japan Society for the Promotion of Science (JSPS) Overseas Research Fellowships.

\section{REFERENCES}

Antoniadis J., et al., 2013, Science, 340, 448

Baron E., Cooperstein J., 1990, ApJ, 353, 597

Bethe H. A., Wilson J. R., 1985, ApJ, 295, 14

Buras R., Rampp M., Janka H.-T., Kifonidis K., 2006, A\&A, 447, 1049

Burrows A., 2013, Reviews of Modern Physics, 85, 245

Demorest P. B., Pennucci T., Ransom S. M., Roberts M. S. E., Hessels J. W. T., 2010, Nature, 467, 1081

Ertl T., Janka H.-T., Woosley S. E., Sukhbold T., Ugliano M., 2016, ApJ, 818, 124

Janka H.-T., Melson T., Summa A., 2016 , Annual Review of Nuclear and Particle Science, 66, 341

Lattimer J. M., Prakash M., 2001, ApJ, 550, 426

Lattimer J. M., Swesty F. D., 1991, Nuclear Physics A, 535, 331

Liebendörfer M., Whitehouse S. C., Fischer T., 2009, ApJ, 698, 1174

Maeder A., Meynet G., 1989, A\&A, 210, 155

Martinez J. G., et al., 2015, ApJ, 812, 143 
Moriya T. J., et al., 2017, MNRAS, 466, 2085

Müller B., Gay D. W., Heger A., Tauris T. M., Sim S. A., 2018, MNRAS, 479, 3675

Nomoto K., 1984, ApJ, 277, 791

Nomoto K., 1987, ApJ, 322, 206

Nomoto K., Hashimoto M., 1988, Phys. Rep., 163, 13

Özel F., Freire P., 2016, ARA\&A, 54, 401

Paxton B., et al., 2015, ApJS, 223, 18

Poelarends A. J. T., Wurtz S., Tarka J., Cole A. L., Hills S. T., 2017, ApJ, 850, 197

Schwab J., Quataert E., Kasen D., 2016, MNRAS, 463, 3461

Shapiro S. L., Teukolsky S. A., 1983, Black holes, white dwarfs, and neutron stars: The physics of compact objects

Siess L., Lebreuilly U., 2018, A\&A, 614, A99

Sukhbold T., Ertl T., Woosley S. E., Brown J. M., Janka H.-T., 2016, ApJ, 821, 38

Sukhbold T., Woosley S. E., Heger A., 2018, ApJ, 860, 93

Summa A., Hanke F., Janka H.-T., Melson T., Marek A., Müller B., 2016, ApJ, 825, 6

Suwa Y., Kotake K., Takiwaki T., Whitehouse S. C., Liebendörfer M., Sato K., 2010, PASJ, 62, L49

Suwa Y., Yoshida T., Shibata M., Umeda H., Takahashi K., 2015, MNRAS, 454, 3073

Suwa Y., Yamada S., Takiwaki T., Kotake K., 2016, ApJ, 816, 43

Suwa Y., Tominaga N., Maeda K., 2017, preprint, (arXiv:1704.04780)

Takahashi K., Yoshida T., Umeda H., 2013, ApJ, 771, 28

Takahashi K., Umeda H., Yoshida T., 2014, ApJ, 794, 40

Takahashi K., Sumiyoshi K., Yamada S., Umeda H., Yoshida T., 2018, submitted to ApJ

Tauris T. M., Langer N., Moriya T. J., Podsiadlowski P., Yoon S.-C., Blinnikov S. I., 2013, ApJ, 778, L23

Tauris T. M., Langer N., Podsiadlowski P., 2015, MNRAS, 451,2123

Timmes F. X., Woosley S. E., Weaver T. A., 1996, ApJ, 457, 834

Toki H., Suzuki T., Nomoto K., Jones S., Hirschi R., 2013, Phys. Rev. C, 88, 015806

Ugliano M., Janka H.-T., Marek A., Arcones A., 2012, ApJ, 757,69

Umeda H., Yoshida T., Takahashi K., 2012, Prog. Theor. Exp. Phys., 01, A302

Woosley S. E., Heger A., 2015, ApJ, 810, 34

Woosley S. E., Weaver T. A., Taam R. E., 1980, in Wheeler C., ed., Type I Supernovae; Proceedings of the Texas Workshop. Austin, TX: Univ. of Texas, pp 96-112

Yoshida T., Suwa Y., Umeda H., Shibata M., Takahashi K., 2017, MNRAS, 471, 4275

\section{APPENDIX A: EVOLUTION OF CO CORES}

We present evolution properties of light $\mathrm{CO}$ cores in which an off-center Ne ignition occurs. Here we show the evolution of CO142 model as an example. Figure A1 shows the evolution of the temperature profile of CO142 model. Figure A2 shows the mass fraction distributions of nine different evolution stages corresponding to the lines $2-10$ shown in Fig. A1.

Carbon ignites at the center for all of the models. An $\mathrm{ONe}$ core (a central region with the $\mathrm{C}$ mass fraction being less than 0.01) forms through convective core $\mathrm{C}$ burning. The line 1 of Fig. A1 shows the temperature profile at $t_{f}-t=1.0 \times 10^{4} \mathrm{yr}$ for CO142 model, where $t_{f}$ is the time at the last step of the calculation. The convective core C burning proceeds for $6.4 \times 10^{3} \mathrm{yr}$. The core grows up through

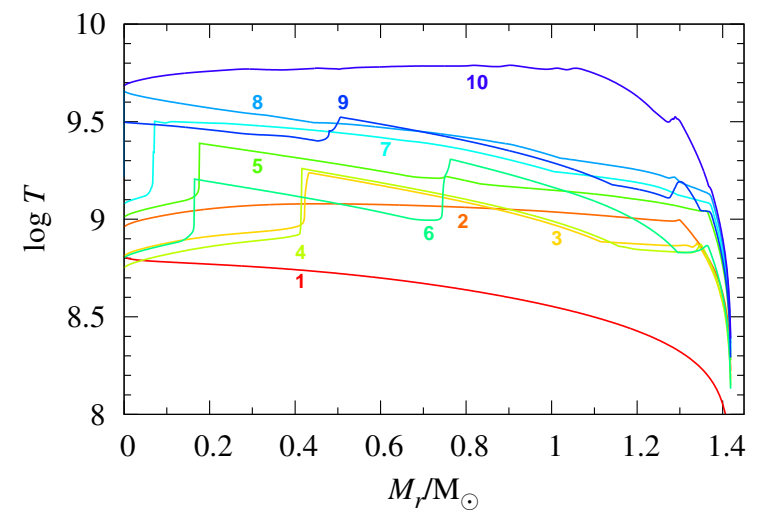

Figure A1. Time evolution of the temperature profile of CO142 model. The evolution numbers correspond to the following: (1) the central C burning at $t_{f}-t=1.0 \times 10^{4} \mathrm{yr},(2)$ the formation of $\mathrm{ONe}$ core at $t_{f}-t=31.7 \mathrm{yr},(3)$ off-center Ne burning at $t_{f}-t=23.5$ $\mathrm{yr}$, (4) off-center O burning at $t_{f}-t=8.9 \mathrm{yr}$, (5) off-center O burning and the growth of the Si layer $t_{f}-t=1.6 \mathrm{yr},(6)$ O-shell burning at $t_{f}-t=1.4 \mathrm{yr},(7)$ off-center Si burning and Fe layer formation $t_{f}-t=4.7 \mathrm{~d},(8)$ the flame arrival at the center and the Fe-core formation at $t_{f}-t=2.8 \mathrm{~d}$, (9) Si-shell burning and Fe core growth at $t_{f}-t=2.4 \mathrm{~d}$, (10) the last profile $\left(t=t_{f}\right)$.

the following several $\mathrm{C}$ shell burnings. The ONe core gradually contracts and the location of the maximum temperature moves outward. The plasma neutrino process dominates in the neutrino energy loss in the temperature inversion region. The line 2 in Fig. A1 shows the temperature profile after the last $\mathrm{C}$ shell burning. Figure A2 $(a)$ shows the mass fraction distribution at that time.

Neon ignites at an off-center region after the ONe core mass becomes $1.32-1.33 \mathrm{M}_{\odot}$. The burning front is formed at the inner edge of the burning layer. The temperature at the burning front becomes the highest after the ignition, while the central temperature decreases (the line 3 of Fig. A1 for CO142 model). The off-center Ne burning makes a large convective $\mathrm{O} / \mathrm{Si}$ layer on the ONe core (Fig. A2(c) for CO142 model). The convective layer extends to $\geq 1 \mathrm{M}_{\odot}$ in the mass coordinate. After the off-center Ne burning, the temperature at the inner edge of the $\mathrm{O} / \mathrm{Si}$ layer decreases. The ONe core contracts again.

Off-center O burning starts at the inner edge of the $\mathrm{O} / \mathrm{Si}$ layer. An inner region of the $\mathrm{O} / \mathrm{Si}$ layer becomes the Si layer and a part of oxygen is burned in the other region. Then, the inner edge of the Si layer gradually moves inward and, thus, the Si layer grows up inward. The energy released in the burning front partially transfers into the core and the oxygen and neon in contact with the burning front are burned into silicon. The line 5 of Fig. A1 and Fig. A2 $(d)$ show the temperature profile and the mass fraction distribution during the off-center O burning for CO142 model. The mass of the ONe core is $\sim 0.2 \mathrm{M}_{\odot}$. The Si-rich layer extends to $\sim 0.9$ $\mathrm{M}_{\odot}$, the $\mathrm{O} / \mathrm{Si}$ layer ranges to $\sim 1.2 \mathrm{M}_{\odot}$, and the $\mathrm{O} / \mathrm{Ne}$ layer remains on the $\mathrm{O} / \mathrm{Si}$ layer.

During the off-center $\mathrm{Ne}$ and $\mathrm{O}$ burnings, the electron fraction in the $\mathrm{O} / \mathrm{Si}$ and $\mathrm{Si}$ layers on the burning front is reduced through electron captures and $\beta^{+}$decays, respectively. The main parent nuclei for electron captures and $\beta^{+}$ decays are ${ }^{26} \mathrm{Al}$ (isomeric state), ${ }^{31} \mathrm{~S}$, and ${ }^{30} \mathrm{P}$. The increase 

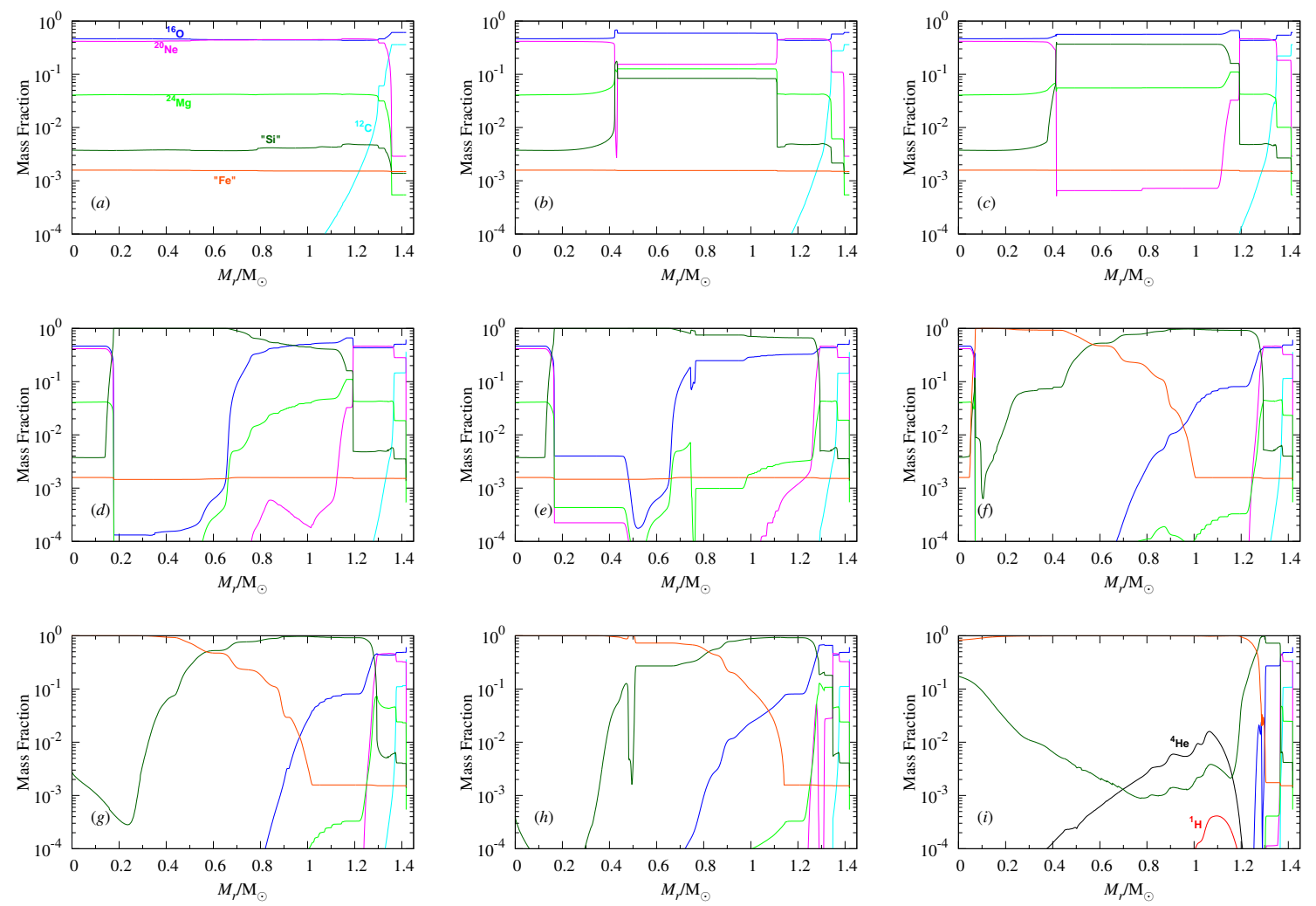

Figure A2. Mass fraction distributions in different evolution stages of CO142 model. Panels $(a)-(i)$ correspond to the evolution number (2)-(10) in Fig. A1.

in the temperature at the burning front enhances these reactions. During the off-center Ne burning of CO142 model, the electron fractions of the $\mathrm{ONe}$ core and the $\mathrm{O} / \mathrm{Si}$ layer are 0.498 and 0.496 , respectively. The electron fraction in the $\mathrm{O} / \mathrm{Si}$ layer becomes smaller than that in the ONe core. Electron captures and $\beta^{+}$decays at the burning front and the electron captures of ${ }^{33} \mathrm{~S}$ and ${ }^{35} \mathrm{Cl}$ in the Si layer reduce the electron fraction in the Si layer. The electron fraction in $M_{r} \sim 0.2-0.5 \mathrm{M}_{\odot}$ reduces to $Y_{e}=0.474$ during the off-center O burning for CO142 model.

Strong $\mathrm{O}$ shell burning occurs after the off-center $\mathrm{O}$ burning. For CO142 model, the $\mathrm{O}$ shell burning extends the region of the Si layer up to $\sim 1.3 \mathrm{M}_{\odot}$ (Fig. A2(e)). This shell burning suppresses the core contraction and the inward motion of the inner edge for a while (the line 6 of Fig. A1 for temperature proflie).

The inner edge of the Si layer continues to move inward and the temperature at the burning front gradually increases. The intermediate elements outside the front are gradually burned to Fe-peak elements. Then, the inner region of the Si layer changes to the Fe layer. The electron fraction in this layer is reduced through electron captures of ${ }^{54} \mathrm{Mn},{ }^{55} \mathrm{Mn}$, and ${ }^{57} \mathrm{Fe}$. The burning front of CO142 model comes to $\sim 0.08 \mathrm{M}_{\odot}$ and the Fe layer grows up to 0.08-0.6 $\mathrm{M}_{\odot}$ at $t_{f}-t=4.7 \mathrm{~d}$ (see the line 7 in Fig A1 for the temperature profile and Fig. A2 $(f)$ for mass fraction distribution). The electron fraction at the inner edge of the Fe layer becomes $\sim 0.453$. The burning front moves inward further and it reaches the center (see the line 8 of Fig. A1 and Fig. A2 $(\mathrm{g})$ for CO142 model). At that time, the central temperature is the highest in the star. The Fe "core" of CO142 model is about $0.6 \mathrm{M}_{\odot}$ and thick Si-rich layer up to $\sim 1.3 \mathrm{M}_{\odot}$ is on the Fe core.

Then, the entire core contracts and the Fe core grows up through several Si shell burnings. The line 9 of Fig. A1 and Fig. A2 $(h)$ show the temperature profile and the mass fraction distribution of CO142 model. During the Si shell burnings, the shell-burning front sometimes becomes the highest temperature. At this time, the Fe core grows up to $\sim 0.8 \mathrm{M}_{\odot}$. The Fe core continues growing until the calculation is terminated. The line 10 of Fig. A1 and Fig. A2(i)) shows the temperature distribution and the mass fraction distribution at the last step of CO142 model. The Fe core has an approximately isothermal structure. The Fe core is surrounded by thin $\mathrm{Si}, \mathrm{Si} / \mathrm{O}, \mathrm{O} / \mathrm{Ne}$, and $\mathrm{O} / \mathrm{C}$ layers.

\section{APPENDIX B: MODIFIED CHANDRASEKHAR LIMIT}

In this section, we give a brief explanation of modified Chandrasekhar mass. From the hydrostatic equation,

$\frac{d P}{d r}=-\frac{G M}{r^{2}} \rho$,

where $P$ is pressure, $r$ is radius, $G$ is the gravitational constant, $M$ is enclosed mass inside $r$, and $\rho$ is density, the dimensionless equation (so-called Lane-Emden equation) is derived as (e.g. Shapiro \& Teukolsky 1983)

$\frac{1}{\xi^{2}} \frac{d}{d \xi}\left(\xi^{2} \frac{d \theta}{d \xi}\right)=-\theta^{N}$ 
Here, the following equations are used:

$\rho=\rho_{c} \theta(\xi)^{N}$

$P=P_{c} \theta(\xi)^{N+1}$,

$r=\alpha \xi$,

$\alpha=\left(\frac{N+1}{4 \pi G} \frac{P_{c}}{\rho_{c}^{2}}\right)^{1 / 2}$,

where $\rho_{c}$ and $P_{c}$ are the central density and pressure, and $N$ is the polytropic index. They are related to each other as $P_{c}=K \rho_{c}^{1+1 / N}$ with a constant $K$. By integrating Eq. (B2) for $\theta(\xi)$ from the center $(\xi=0)$ toward the stellar surface ( $\xi_{N}$ where $\theta\left(\xi_{N}\right)=0$ ) with boundary conditions, $\theta(0)=1$ and $\theta^{\prime}(0)=1$, the stellar structure is determined. From the solution the stellar radius and mass are given by

$$
\begin{aligned}
R & =\alpha \xi_{N}=\left(\frac{N+1}{4 \pi G} \frac{P_{c}}{\rho_{c}^{2}}\right)^{1 / 2} \xi_{N}, \\
M & =\int_{0}^{R} 4 \pi \rho r^{2} d r=4 \pi \alpha^{3} \rho_{c} \int_{0}^{\xi_{N}} d \xi \xi^{2} \theta^{N} \\
& =-\left.4 \pi \alpha^{3} \rho_{c}\left(\xi^{2} \frac{d \theta}{d \xi}\right)\right|_{\xi=\xi_{N}} .
\end{aligned}
$$

By making use of

$\varphi_{N}=-\left.(N+1)^{3 / 2}\left(\xi^{2} \frac{d \theta}{d \xi}\right)\right|_{\xi=\xi_{N}}$,

the mass is given by

$M=\left(\frac{1}{4 \pi G^{3}} \frac{P_{c}^{3}}{\rho_{c}^{4}}\right)^{1 / 2} \varphi_{N}$.

$\varphi_{N}$ depends on $N$. For instance, $N=3$ gives 16.15 and $N=$ 3.3 gives 17.27. The equation of state for relativistic electrons is given by $P=K\left(\rho Y_{e}\right)^{4 / 3}$, where $Y_{e}$ is the electron fraction and $K=\frac{\left(3 \pi^{2}\right)^{1 / 3}}{4} \frac{\hbar c}{m_{N}^{4 / 3}}$ with $\hbar, c$ and $m_{N}$ being reduced Planck constant, speed of light and nucleon mass, respectively. If $Y_{e}$ is constant, it corresponds to $N=3$. However, during the stellar evolution, $Y_{e}$ decreases due to the electron capture. The central value is approximately $Y_{e, c} \sim 0.42$ when $\rho_{c} \sim$ $10^{10} \mathrm{~g} \mathrm{~cm}^{-3}$. Assuming $Y_{e, c} \propto \rho^{\alpha}$ and $Y_{e, c}=0.5$ for $\rho_{c}=10^{7}$ $\mathrm{g} \mathrm{cm}^{-3}$, which indicates $\alpha \approx-0.025$, we get $P_{C} \propto \rho_{c}^{\frac{4}{3}(1+\alpha)} \sim$ $\rho_{c}^{1+\frac{1}{3.3}}$, thus $N \sim 3.3$.

In addition to the degeneracy pressure of electrons, the finite temperature correction is also important for the presupernova cores. Thus, the pressure is given by (Baron \& Cooperstein 1990)

$P=K\left(\rho Y_{e}\right)^{4 / 3}\left[1+\frac{2}{3}\left(\frac{s_{e}}{\pi Y_{e}}\right)^{2}\right]$,

where $s_{e}$ is the electronic entropy.

Combining Eqs. (B10) and (B11), we get

$$
\begin{aligned}
M & =1.09 M_{\odot}\left(\frac{Y_{e, c}}{0.42}\right)^{2}\left[1+\frac{2}{3}\left(\frac{s_{e, c}}{\pi Y_{e, c}}\right)^{2}\right]^{3 / 2} \\
& \approx 1.09 M_{\odot}\left(\frac{Y_{e, c}}{0.42}\right)^{2}\left[1+\left(\frac{s_{e, c}}{\pi Y_{e, c}}\right)^{2}\right]
\end{aligned}
$$

where $Y_{e, c}$ and $s_{e, c}$ are the central values of $Y_{e}$ and $s_{e}$. Here we use $\varphi_{N}=17.27$ (corresponding to $N=3.3$ ), since we are now interested in the stellar structure in which the electron capture reduces $Y_{e}$. We also assume that the thermal correction (the second term in the square brackets) is much smaller than unity.

This paper has been typeset from a $\mathrm{T}_{\mathrm{E}} \mathrm{X} / \mathrm{LAT} \mathrm{E}$ file prepared by the author. 